\title{
Quantitative Estimation of Ising-type Magnetic Anisotropy in a Family of $\mathrm{C}_{3}$ Symmetric Co"l Complexes
}

\author{
Amit Kumar Mondal, ${ }^{a}$ Jesús Jover, ${ }^{b}$ Eliseo Ruiz ${ }^{* b}$ and Sanjit Konar*a
}

\begin{abstract}
In this paper, the influence of the structural and chemical effects on the Ising-type magnetic anisotropy of penta-coordinate Co" complexes has been investigated using a combined experimental and theoretical approach. For this, a deliberate design and synthesis of four penta-coordinate $\mathrm{Co}^{\prime \prime}$ complexes [ $\left.\mathrm{Co}(\mathrm{TPA}) \mathrm{Cl}\right] \cdot \mathrm{ClO}_{4}(\mathbf{1}),[\mathrm{Co}(\mathrm{TPA}) \mathrm{Br}] \cdot \mathrm{ClO}_{4}(2)$, [ $\left.\mathrm{Co}(\mathrm{tbta}) \mathrm{Cl}\right] \cdot\left(\mathrm{ClO}_{4}\right) \cdot(\mathrm{MeCN})_{2} \cdot\left(\mathrm{H}_{2} \mathrm{O}\right)(3)$ and $[\mathrm{Co}(\mathrm{tbta}) \mathrm{Br}] \cdot \mathrm{ClO}{ }_{4}(4)$ using the tripodal ligands tris(2-methylpyridyl)amine (TPA) and tris[(1-benzyl-1H-1,2,3-triazole-4-yl)methyl]amine) (tbta) have been carried out. Detailed dc and ac measurements show the existence of field induced slow magnetic relaxation behaviour of Co" centres with Ising-type magnetic anisotropy. A quantitative estimation of ZFS parameters has been effectively achieved using detailed ab initio theory calculations. Computational studies reveal that the wavefunction of all the studied complexes has a very strong multiconfigurational character that stabilizes the largest $M_{s}= \pm 3 / 2$ components of the quartet state and hence produce a large negative contribution to ZFS parameters. The difference between the magnitudes of the Ising-type anisotropy can be explained via ligand field theory considerations, i.e. $D$ is larger and negative in the case of weak equatorial $\sigma$-donating and strong apical $\pi$-donating ligands; therefore providing an implement to forecast the magnetic anisotropy in penta-coordinate Co" complexes. In order to elucidate the role of intermolecular interactions in the magnetic relaxation behavior between adjacent Co" centres, a diamagnetic isostructural $\mathrm{Zn}^{\prime \prime}$ analogue (5) was synthesized and the magnetic behaviour was examined.
\end{abstract}

\section{Introduction}

Single-molecule magnets (SMMs), a kind of molecular nanomagnets that show slow relaxation of the magnetization and magnetic hysteresis below the blocking temperature $\left(T_{\mathrm{B}}\right)$, have attracted a lot of research interest over the last two decades. ${ }^{[1]}$ The magnetism of SMMs originates from an energy barrier $(U)$ that precludes reversal of the magnetization at low temperatures that is a result of the combined effect from the ground-state spin and magnetic anisotropy of the molecule $\left[U=|D| S^{2}\right.$ and $U=|D|$ $\left(S^{2}-1 / 4\right)$ for integer and non-integer $S$, respectively]. ${ }^{[2]}$ The first generation of SMMs were ferro-magnetically coupled transition metal clusters which have a large spin ground state along with a large Ising-type anisotropy. ${ }^{[1 b, d]}$ Nevertheless, the control of the anisotropy in cluster systems was very difficult, and the increase in total spin of these systems led to a decrease in the total anisotropy in most of the cases. In the last decade, extensive efforts have been dedicated towards the synthesis of a new class of mononuclear SMMs that are commonly referred to as single-ion magnets (SIMs), where the SMM-like behavior comes from only one paramagnetic center. While most of the reported SIMs are based on the lanthanide complexes, ${ }^{[3]}$ the search for new SIMs has been extended to transition-metal based complexes. ${ }^{[4-5]}$ Low-coordinate metal centres are commonly

[a] A. K. Mondal, Prof. Dr. S. Konar

Department of Chemistry, Indian Institute of Science Education and Research Bhopal, Bhopal Bypass road, Bhauri, Bhopal 462066, MP (India). Tel: (+91) 755-6691313. Fax: (+91) 7556692392.

E-mail: skonar@iiserb.ac.in

[b] Dr. J. Jover, Prof. Dr. E. Ruiz

Departament de Química Inorgànica I Orgànica, Secció de

Química Inorgànica and Institut de Recerca de Química Teòrica i

Computacional, Universitat de Barcelona, Diagonal 645, E-08028

Barcelona, Spain.

E-mail: eliseo.ruiz@qi.ub.es observed among the reported transition-metal based SIMs, where the single ion anisotropy is enhanced because of the partially unquenched orbital angular momentum. ${ }^{[6]}$ Among transition metals, Co" based complexes are mostly attractive candidates for designing SIMs, since they have a non-integer spin ground state, ${ }^{[7]}$ which decreases the possibility of quantum tunnelling of magnetization (QTM) ${ }^{[8]}$

Mononuclear penta-coordinate Co" SMMs have been reported to typically display trigonal bipyramidal (TBY) or square pyramidal $(\mathrm{SPY})^{[\mathrm{kk}-\mathrm{m}]}$ coordination geometries and many attempts have aimed to rationally control the single ion anisotropy in recent years. ${ }^{[4]}$ However, the elements regulating single ion anisotropy are not well comprehended and intricate control over magnetic anisotropy remains still a challenging task. For transition metal complexes, an axial symmetry imposed by the ligands is usually an essential requirement to obtain large magnetic anisotropies. Herein, we report the synthesis of four different $\mathrm{Co}^{\text {"l }}$ complexes bearing the tripodal ligands TPA and tbta $($ TPA $=\operatorname{tris}(2-$ methylpyridyl)amine and tbta $=\operatorname{tris}[(1-$ benzyl-1 $\mathrm{H}-1,2,3$-triazole-4$\mathrm{yl}$ )methyl]amine) that were anticipated to impose $C_{3 v}$ axial symmetry in the resulting complexes. The dynamic magnetization behaviour of penta-coordinate $\mathrm{Co}^{\text {"l }}$ complexes [Co(TPA)Cl] $\cdot \mathrm{ClO}_{4} \quad$ (1), $\quad[\mathrm{Co}(\mathrm{TPA}) \mathrm{Br}] \cdot \mathrm{ClO}_{4}$ [Co(tbta) $\mathrm{Cl}] \cdot\left(\mathrm{ClO}_{4}\right) \cdot(\mathrm{MeCN})_{2} \cdot\left(\mathrm{H}_{2} \mathrm{O}\right)(3)$ and $[\mathrm{Co}(\mathrm{tbta}) \mathrm{Br}] \cdot \mathrm{ClO}_{4}(4)$ have been studied and the influence of structural and chemical effects on the magnitude of the magnetic anisotropy have been investigated using a combined experimental and theoretical approach. The detailed $a b$ initio theory calculations disclose that the wavefunction of complexes 1-4 has a very strong multiconfigurational character that stabilizes the largest $M_{s}=$ $\pm 3 / 2$ components of the quartet state and hence produce a large negative contribution to $D$ parameters. 


\section{Results and Discussion}

\section{Structural description of complexes 1 - 4:}

Single-crystal $X$-ray analysis of 1 and 2 reveals that the complex 1 crystallizes in the monoclinic $P 2{ }_{1} / \mathrm{c}$ space group and complex 2 crystallizes in the triclinic $P-1$ space group (Table S1). The molecular structures of the complexes are shown in Figure 1.

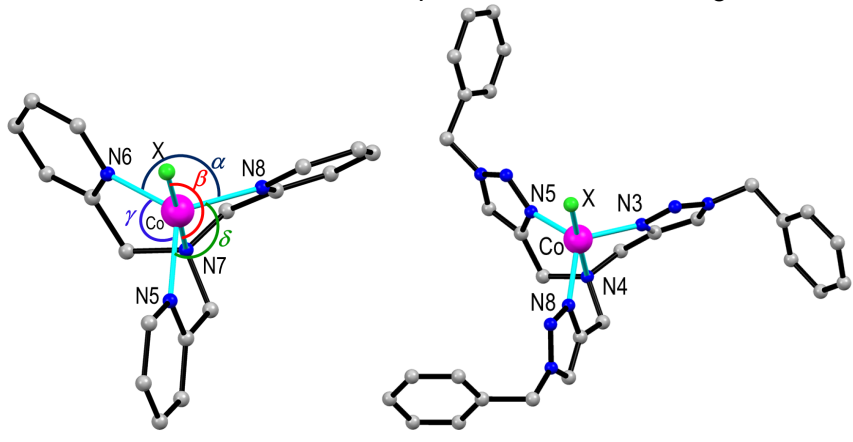

Figure 1. View of the molecular structures for complexes 1 and 2 (left), where $\mathrm{X}=\mathrm{Cl}(\mathbf{1})$ and $\mathrm{X}=\mathrm{Br}(2)$. The bond angles $(\alpha, \beta, \gamma$ and $\delta)$ around $\mathrm{Co}^{\prime \prime}$ centres have been emphasized in this figure (left); View of the molecular structures for complexes 3 and 4 , where $X=\mathrm{Cl}(3)$ and $X=\mathrm{Br}(\mathbf{4})$ (right); hydrogen atoms are omitted for clarity.

The tripodal ligand coordinates in a tetradentate fashion and the geometry at the Co" centre is distorted trigonal bipyramidal. The ligands (TPA) are dispersed at the apices of a trigonal bipyramid with crystallographic $C_{3}$ symmetry; the three equatorial sites are employed by the terminal amines and the axial sites are occupied by the central amine and the chloride ion. In complex 1 the largest angles within the four atoms N6, N7, N8 and Cl2 are $\beta=178.64(2)^{\circ}$ for N7-Co3-Cl2, and $\alpha=118.67(1)^{\circ}$ for N8Co3-N6 (Table S2). Thus, $\tau$ is $(178.64-118.67) / 60=0.999 .^{[9]}$ This indicates that the geometry around $\mathrm{Co}^{\prime \prime}$ is highly distorted trigonal bipyramidal. Additionally, a modified index parameter, $\chi$, has been proposed to describe the trigonality of five-coordinate complexes, where $\chi=(\beta+\gamma+\delta-2 \alpha) / 180(\gamma$ and $\delta$ represent the remaining bond angles around the metal ion without the donor atoms defining $\beta$ ). The $\chi$ value for 1 is 0.937 , which suggests that the geometry around $\mathrm{Co}^{\prime \prime}$ is better described as a distorted trigonal bipyramidal (Figure S1). The Co" ion lies $0.449 \AA$ below the equatorial plane of the three nitrogen atoms in complex 1 (0.455 $\AA$ in complex 2). The Co-N axial bond length (2.201(1) $\AA$ ) is slightly longer than the equatorial ones $(2.056(2)-2.069(1) \AA$ ). In complex 1, the Co- $\mathrm{Cl}$ distance is equal to 2.283(1) $\AA$ whereas in 2 the $\mathrm{Co}-\mathrm{Br}$ distance is equal to 2.431(1) $\AA$. Additional SHAPE analysis confirms that the five-coordinate Co" centres adopt geometries which can be better described as distorted (a)

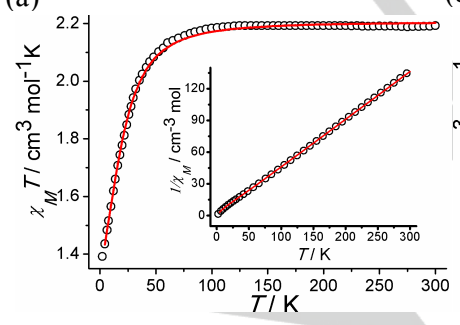

(b)

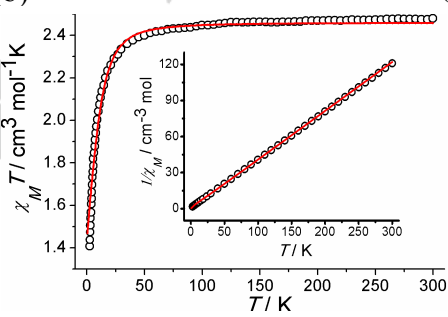

trigonal bipyramidal, with minimum CShM values of 1.880 and 2.298 for 1 and $\mathbf{2}$ respectively (Table S4). In both the complexes, there are significant $\pi$-interaction and intermolecular hydrogenbonding network, which supports the formation of a supramolecular arrangement (Figures S2-S3 and Table S6-S7). In complex 1, hydrogen atoms of pyridyl rings are involved in intermolecular hydrogen bonding (Table S4) with chloride atoms and perchlorate molecules and resulted in the formation of a supramolecular two dimensional arrangement (Figure S2). In addition to the $\mathrm{H}$-bonding interactions, strong $\mathrm{CH} \cdots \pi$ interactions are also noticed with $\mathrm{CH}$ to centroid distances of 3.598(3) $\AA$ and 3.346(5) A for 1 and 2, respectively.

Single-crystal X-ray analysis of $\mathbf{3}$ and $\mathbf{4}$ showed that complexes 3 and 4 crystallize in the monoclinic $P 2{ }_{1} / \mathrm{n}$ and cubic $P a-3$ space groups respectively (Table S1). The Coll center is coordinated by the three nitrogen atoms of triazole rings of tbta ligand. The bond distance between the cobalt center and the central amine nitrogen of tbta is 2.344(1) $\AA$, which is longer than the terminal ones (2.037(2) - 2.049(1) $\AA$ ). In complex 3, the Co-Cl distance is equal to 2.260(1) $\AA$ whereas in $\mathbf{4}$ the $\mathrm{Co}-\mathrm{Br}$ distance is equal to 2.402(1) $\AA$ (Table S3). The Co" center lies $0.531 \AA$ above the plane containing the three triazole nitrogen-donor atoms in complex 3 (0.514 $\AA$ in complex 4). Both the complexes are involved in intermolecular hydrogen bonding (Table S8-S9) with the lattice solvent molecules and perchlorate anions and these interactions support the formation of supramolecular two dimensional arrangement (Figures S4-S5).

Some important structural features for complexes 1-4 have been summarized in table S5. For comparison, the Co-N distances to the pyridine rings of TPA ligands in complex $\mathbf{1}$ and $\mathbf{2}$ are slightly longer than the Co- $\mathrm{N}_{\text {(triazole) }}$ distances observed in the case of 3 and 4. The main difference, however, is the Co- $\mathrm{N}_{(a m i n e)}$ distance, which is 2.344(1) $\AA$ for complex 3, whereas for $\mathbf{1}$ the corresponding distance is 2.201(1) $\AA$. Therefore, the central amine nitrogen binds much more strongly to the Co" center in 1 as compared to 3 and hence the ligand TPA is coordinated to the $\mathrm{Co}$ " center in a more compact manner. ${ }^{[10]}$

\section{Magnetic Property Studies:}

The purity of the as-synthesized products is demonstrated by the good agreement of the bulk phase powder X-ray diffraction patterns with the simulated ones (Figure S6-S7). Magnetic susceptibility measurements have been carried out under direct current (DC) and an applied field of $0.1 \mathrm{~T}$. At room temperature, $\chi_{w} T$ values $\left(\chi_{*}=\right.$ molar magnetic susceptibility) of 2.29 (1), 2.17 (2), 2.53 (3) and $2.47(4) \mathrm{cm}^{3} \mathrm{~K} \mathrm{~mol}^{-1}$ have been obtained, which are larger than the spin-only value of $1.875 \mathrm{~cm}^{3} \mathrm{~mol}^{-1} \mathrm{~K}$ for a high-spin $\mathrm{Co}^{\text {Il }}$ ion. These values fall well in the range of 2.1-3.4 $\mathrm{cm}^{3} \mathrm{~mol}^{-1} \mathrm{~K}$ for the highly anisotropic Coll ions with a significant

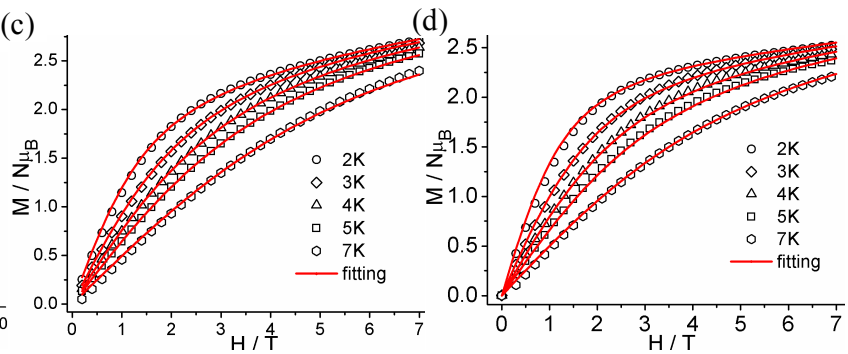

Figure 2. $X_{M} T$ vs. $T$ plots measured at $0.1 \mathrm{~T}$ for complex 2 (a) and 4 (b). 1/ $X_{M} v s . T$ plots shown in the inset; $M / N \mu_{B} v s . H$ plots for complexes 2 (c) and 4 (d) at the indicated temperatures. The red lines correspond to those obatined with the best fit. 
(a)

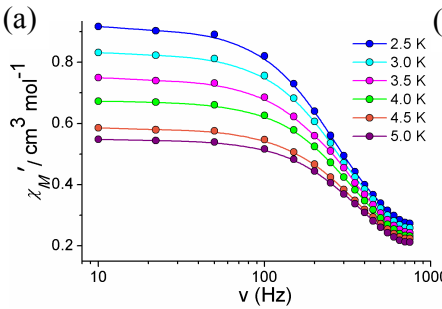

(b)

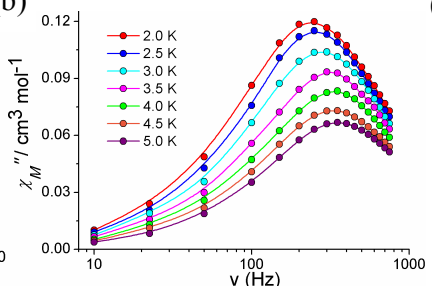

(c)

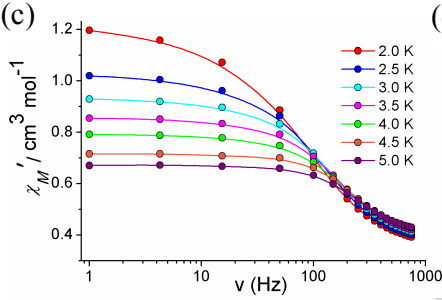

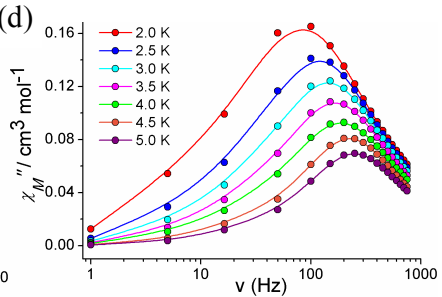

Figure 3. Frequency dependency of the in-phase $\left(X_{M}\right)(a)$ and out-of-phase $\left(X_{M}{ }^{\prime \prime}\right)$ (b) AC magnetic susceptibility plots for complex 2; Frequency dependency of the in-phase $\left(X_{M}{ }^{\prime}\right)(c)$ and out-of-phase $\left(X_{M}{ }^{\prime \prime}\right)(d)$ AC magnetic susceptibility plots for complex 4 at 1000 Oe.

orbital contribution. ${ }^{[11]}$ Upon cooling from room temperature, the $\chi_{w} T$ values remain constant down to $70 \mathrm{~K}$, below which they collapse, reaching values of $1.48(1), 1.39(2), 1.65(3)$ and 1.40 (4) $\mathrm{cm}^{3} \mathrm{~mol}^{-1} \mathrm{~K}$ at $2 \mathrm{~K}$ (Figure 2 and S8). The decline of $\chi_{\mathrm{u}} T$ curves at low temperature can be attributed to intrinsic magnetic anisotropy of the Coll ions. Magnetization data $\left(M / N \mu_{\mathrm{B}}\right.$ vs. $\left.H\right)$ have been collected and reach the highest values of 2.67 (1), $2.71(2), 2.61$ (3) and $2.52(4) N \mu_{\mathrm{B}}$ at $2 \mathrm{~K}$ and $7 \mathrm{~T}$ (Figure 2 and S8). These values are well below the theoretical saturation for an $S=3 / 2$ system $\left(M_{\text {sat }}=3.3\right.$ with $\left.\mathrm{g}=2.2\right)$. The magnetization values do not saturate even at the highest available fields and the $M / N \mu_{\mathrm{B}}$ vs. $H / T$ plots display that all isotherm magnetization plots do not fall on the same master curve signifying the highly magnetic anisotropic systems (Figure S9). The spin Hamiltonian shown eqn (1) is used to qualitatively define the magnetic anisotropy

$H=g \mu_{\mathrm{B}} S \cdot B+D\left[S_{\mathrm{z}}{ }^{2}-S(S+1) / 3\right]+E\left(S_{x}{ }^{2}-S_{y}{ }^{2}\right)$

where $\mu_{\mathrm{B}}, S$ and $B$ represent the Bohr magneton, spin $(S=3 / 2$ for 1-4), and magnetic field vectors, respectively; $D$ and $E$ terms represent the single-ion axial and rhombic ZFS parameters. The $\mathrm{PHI}$ code $^{[12]}$ has been employed to quantify the anisotropy parameters of the Coll centres by concomitant fitting of the $\chi_{u} T$ vs. $T$ and $M / \mathrm{N} \mu_{\mathrm{B}}$ vs. $H$ plots; during the fitting procedure the $g$ tensor has been assumed to be isotropic. The best fits of the reduced magnetization data give $D=-10.1(2) \mathrm{cm}^{-1},|E|=1.8(4)$ $\mathrm{cm}^{-1}$, and $\mathrm{g}=2.28$ for $1 ; D=-7.8(7) \mathrm{cm}^{-1},|E|=2.1(3) \mathrm{cm}^{-1}$, and $\mathrm{g}=2.23$ for $2 ; D=-7.5(4) \mathrm{cm}^{-1},|E|=0.4(6) \mathrm{cm}^{-1}$, and $\mathrm{g}=2.24$ for 3; $D=-4.3(8) \mathrm{cm}^{-1},|E|=0.03(2) \mathrm{cm}^{-1}$, and $\mathrm{g}=2.21$ for 4 . Similar quality fits have been obtained starting from both positive and negative $E$ parameters, demonstrating that these fits are not sensitive to the sign of $E$ parameter. The results of $D, E$ and $g$ values of 1-4 agree well with the values obtained from EPR studies of similar trigonal bipyamidal Co" complexes reported by Gatteschi et al..$^{[13]}$

The negative zero-field splitting parameters $(D)$ for complexes 14 indicate the possibility of exhibiting SMM behaviour. To probe the relaxation dynamics of 1-4, AC magnetic susceptibility measurements were carried out in the temperature range of 1.8$10 \mathrm{~K}$ at a $3.5 \mathrm{Oe}$ ac field. No out-of-phase ac susceptibility $\left(\chi_{\mathrm{M}}{ }^{\prime \prime}\right)$ signal was observed for them under a zero dc field. This can be ascribed to the presence of quantum tunnelling of the magnetization (QTM) through the thermal relaxation barrier between the degenerate ground $\pm 3 / 2$ levels. For a non-integer spin system with $D<0$, transverse anisotropy cannot stimulate the QTM process through mixing of the wave functions corresponding to the \pm Ms levels owing to parity effects; ${ }^{[8]}$ and thus, the quantum tunnelling is possibly due to hyperfine and dipolar arbitrated relaxation processes. ${ }^{[14]}$ The application of a $1000 \mathrm{Oe}$ dc field splits the energy of the \pm Ms doublets, supresses the QTM relaxation pathway and all complexes show frequency-dependent ac signals typically observed for fieldinduced 3d-SIM species (Figure 3 and S10). The Cole-Cole plots (Figure 4 and S11) have been generated from the frequency-dependent ac susceptibility data. The fit of the $\chi_{M}$ " vs $\chi_{M}{ }^{\prime}$ data at each temperature using the generalized Debye model ${ }^{[15]}$ produces the corresponding $\alpha$ values. This parameter determines the width of the distribution of relaxation times, so that $\alpha=1$ corresponds to an infinitely wide distribution of relaxation times. In contrast, $\alpha=0$ indicates a relaxation with a single time constant. Values within the ranges 0.02-0.21 (1), 0.06-0.24 (2), 0.07-0.26 (3) and 0.04-0.30 (4), have been obtained for our complexes, suggesting in all cases a narrow distribution of the relaxation time. The effective energy barrier $\left(U_{\text {eff }}\right)$ and relaxation times $\left(\tau_{0}\right)$ have been determined using the Arrhenius eqn (2): ${ }^{[16]}$

$\ln (1 / \tau)=\ln \left(1 / \tau_{0}\right)-U_{\text {eff }} / k T$

where $k$ is the Boltzmann constant and $1 / \tau_{0}$ is the preexponential factor. A linear fit according to Arrhenius equation produces $U_{\text {eff }}=12 \mathrm{~cm}^{-1}$ and $\tau_{0}=7.2 \times 10^{-6} \mathrm{~s}$ for $1 ; U_{\text {eff }}=8.7$ $\mathrm{cm}^{-1}$ and $\tau_{0}=5.8 \times 10^{-6} \mathrm{~s}$ for $2 ; U_{\text {eff }}=8.1 \mathrm{~cm}^{-1}$ and $\tau_{0}=3.5 \times$ $10^{-6} \mathrm{~s}$ for 3; $U_{\text {eff }}=5 \mathrm{~cm}^{-1}$ and $\tau_{0}=2.1 \times 10^{-6} \mathrm{~s}$ for 4 (Figure 4 and S11). The $\tau_{0}$ values are at the higher end of the experimental range found for $\mathrm{SMMs}^{[17 \mathrm{a}, \mathrm{b}]}$ and are similar to those found for other Co" SIMs. ${ }^{[17 \mathrm{c}]}$ This strongly suggests that the quantum pathway of relaxation at very low temperatures is not fully suppressed by the effects of the applied dc field. The thermally activated relaxation process at the high-temperature regime specifies that an Orbach pathway ${ }^{[18]}$ through the excited $M s= \pm 1 / 2$ level should be followed. In this regime, the system is excited to the $M s= \pm 1 / 2$ level by absorption of phonons followed by an emission of phonons to reach the Ms $= \pm 3 / 2$ ground state ${ }^{[19]}$ The energy gap between the $M s= \pm 1 / 2$ and $M s= \pm 3 / 2$ levels is given by $\left(D^{2}+3 E^{2}\right)^{1 / 2}$. Considering the $D$ values resulting from the fitting of the magnetization data, the effective energy barriers $\left(U_{\text {eff }}\right)$ are obtained: $20.2(1), 15.6(2), 15.0(3)$ and 8.6 (4) $\mathrm{cm}^{-1}$. Normally, these values are larger than the $U_{\text {eff }}$ values, obtained from ac susceptibility measurements, this is probably due to the existence of a substantial quantum relaxation 
(a)

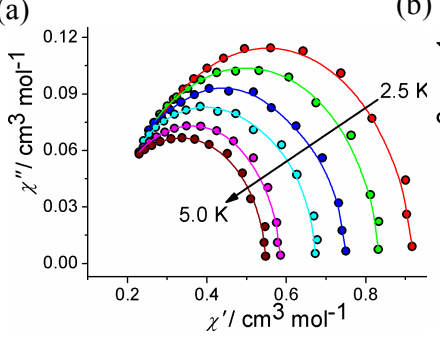

(b)

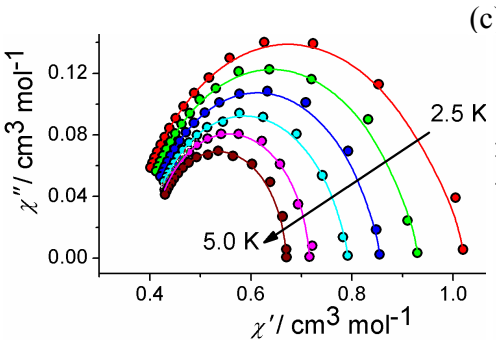

(c)

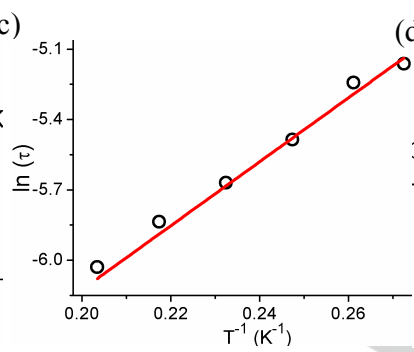

(d) 4.8

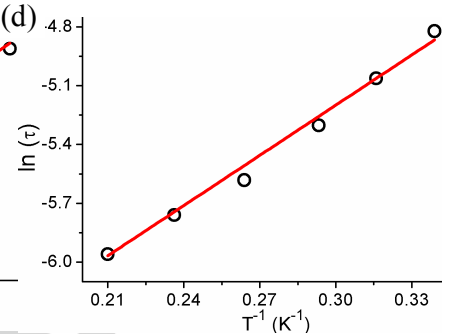

Figure 4. Cole-Cole plots for complex 2 (a) and 4 (b). Solid lines represent the best fit; In $(\tau)$ vs. $T^{-1}$ plots for complex 2 (c) and 4 (d). The red lines are the best fit of the Arrhenius equation.

pathway which is not fully supressed by the applied dc field. The single-phonon direct process and the Raman process may have also a considerable contribution to the spin relaxation behaviour. On a comparative point of view, the difference in the magnitude of $D$ between 1 and 2 can be explained via ligand field theory. The $\mathrm{Co}-\mathrm{Cl}$ distance in $1(2.283 \AA)$ is shorter than the $\mathrm{Co}-\mathrm{Br}$ distance in 2 (2.431 $\AA$ ), as a consequence $\mathrm{Br}^{-}$is found to have a weaker $\pi$-donating effect than $\mathrm{Cl}^{-}$. That results in a higher stabilization of the $d_{x y} / d_{x 2-y 2}$ pair of orbitals for complex 1 , which in the end produces a smaller energy gap between occupied and semioccupied orbitals and therefore a higher $|D|$ value. This behaviour should be also expected for complexes with the tbta ligand (3 and 4). Furthermore, the average Co-N bond distance in the equatorial plane is larger for 1 (2.061 $\AA$ ) than for 3 (2.042 $\AA$ ). Therefore, the $\sigma$-donor effect of the equatorial amine nitrogen atoms is larger for 3 than for 1 and the $d_{x y} / d_{x 2-y 2}$ pair of orbitals is higher in energy in the former than in the latter. This produces a larger energy gap between occupied and semioccupied orbitals for complex 3 (the computed energy gaps are 2796.3 and $3601.8 \mathrm{~cm}^{-1}$ for complexes 1 and 3 , respectively) and hence $|D|$ is likely to be smaller in 3 . As a whole, it should be expected that weak equatorial $\sigma$-donating and strong apical $\pi$-donating ligands would produce larger Ising-type anisotropy; providing an implement to forecast the single-ion magnetic anisotropy in trigonal bipyramidal Co" complexes.

In order to investigate the effect of inter- and intramolecular exchange on the magnetic behavior, we have studied the effect of magnetic dilution on relaxation of the magnetization. In order to do this we have synthesized the diamagnetic isostructural $\mathrm{Zn}^{\prime \prime}$ analogue (5) (see ESI for experimental and X-ray details, Figure S12 and Table S10). After that, we have prepared the doped sample in which the Co" complex (3) has been magnetically diluted with the $\mathrm{Zn}^{\prime \prime}$ analogue in a 5:95 percentage ratio. AC susceptibility measurements have been carried out on a polycrystalline sample of the diluted complex (Figure S13 (left)). In this case the effective energy barrier $\left(U_{\text {eff }}\right)$ and relaxation time $\left(\tau_{0}\right)$ have been determined using the Arrhenius equation; the bset fit provides $U_{\text {eff }}=7.5 \mathrm{~cm}^{-1}$ and $\tau_{0}=3.2 \times 10^{-6} \mathrm{~s}$ (Figure $\mathrm{S} 13$ (right)). Therefore, the relaxation energy barriers show no significant difference between the diluted sample and the undiluted one. In view of this, we may conclude that intermolecular forces and dipolar interactions are negligible; and therefore, the relaxation processes in the case of the studied mononuclear Co" complexes are of single ion origin.

\section{Ab initio calculations}

The magnetic properties of the low-lying states of complexes 1-4 were analyzed by means of an $a b$ initio multireference methodology; the computed second-order anisotropy parameters and excitation energies for all the compounds are collected in Table 1. These values have been obtained from two different electronic structure calculations that have been carried out with the ORCA ${ }^{[20]}$ and MOLCAS $8.0^{[21-23]}$ software packages. ORCA produces two sets of results: CASSCF and CASSCF + NEVPT2 (which is the method of choice to introduce the dynamic correlation effects), both including spin-orbit contributions. On the other hand MOLCAS has been only able to provide CASSCF results, including spin-orbit effects that have been introduced with the SO-RASSI method. Additional CASPT2 calculations for all the complexes were also carried out but they showed serious convergence problems and were thus finally discarded.

The different computational methods employed produce similar values for most of the anisotropy parameters of the studied Co" complexes, although the inclusion of the dynamic correlation effects with NEVPT2 tends to produce slightly lower values. The computed $D,|E|$ and $g_{\text {iso }}$ values are similar to those obtained in the experimental fit (Table 1). In all cases, a $3 / 2$ ground state is found for complexes 1-4 before including the spin-orbit effects. In these conditions, the lowest-lying spin-orbit free excited states are found at relatively high energies ca. $3200-5000 \mathrm{~cm}^{-1}$ above the ground state ( $\delta$ in Table 1).

Table 1. ORCA/CASSCF, ORCA/CASSCF + NEVPT2, and MOLCAS/CASSCF + RASSI computed $D,|E|$, and giso values for the ground state of complexes 1-4. $\delta$ and $\Delta$ are the computed first excitation energies before and after including the spin-orbit effects, respectively. The $\Delta$ value corresponds to the energy difference between the ground and the first excited Kramers' doublets.

\begin{tabular}{|c|c|c|c|c|c|c|}
\hline Complex & $\begin{array}{r}D_{\text {expt }} \\
\left(\mathrm{cm}^{-1}\right)\end{array}$ & $\begin{array}{c}D_{\text {calc }} \\
\left(\mathrm{cm}^{-1}\right)\end{array}$ & $\begin{array}{c}|E| \\
\left(\mathrm{cm}^{-1}\right)\end{array}$ & $\begin{array}{c}\delta \\
\left(\mathrm{cm}^{-1}\right)\end{array}$ & $\begin{array}{c}\Delta \\
\left(\mathrm{cm}^{-1}\right)\end{array}$ & giso \\
\hline $1^{a}$ & & -9.0 & 2.0 & 3219.7 & 19.2 & 2.27 \\
\hline $1^{b}$ & $-10.1(2)^{d}$ & -6.0 & 1.6 & 4458.1 & 13.3 & 2.20 \\
\hline $1^{\mathrm{c}}$ & & -9.5 & 2.0 & 3281.6 & 20.2 & 2.27 \\
\hline $2^{\mathrm{a}}$ & & -7.0 & 1.7 & 3215.2 & 15.3 & 2.27 \\
\hline $2^{\mathrm{b}}$ & $-7.8(7)^{d}$ & -4.4 & 1.3 & 4471.2 & 10.0 & 2.20 \\
\hline $2^{c}$ & & -7.5 & 1.8 & 3268.7 & 16.1 & 2.27 \\
\hline $3^{\mathrm{a}}$ & & -8.3 & 0.5 & 3692.7 & 16.7 & 2.26 \\
\hline $3^{\mathrm{b}}$ & $-7.5(4)^{d}$ & -6.3 & 0.4 & 5006.2 & 12.6 & 2.20 \\
\hline $3^{\mathrm{C}}$ & & -8.8 & 0.5 & 3728.0 & 17.7 & 2.27 \\
\hline $4^{\mathrm{a}}$ & & -6.0 & 0.0 & 3619.6 & 12.0 & 2.27 \\
\hline $4^{b}$ & $-4.3(8)^{d}$ & -4.4 & 0.0 & 4904.5 & 8.9 & 2.20 \\
\hline $4^{c}$ & & -6.3 & 0.0 & 3654.5 & 12.7 & 2.28 \\
\hline
\end{tabular}

${ }^{a}$ ORCA/CASSCF. ${ }^{b}$ ORCA/CASSCF + NEVPT2. ${ }^{\circ}$ MOLCAS/CASSCF + RASSI. ${ }^{d}$ Experimental values. 
Once the spin-orbit effects are included a set of Kramers' doublets (KDs, $\Delta$ ) for each complex is obtained. In all cases the first $K D$ is found at a very low energy, typically below $20 \mathrm{~cm}^{-1}$, and may actively participate in the spin relaxation processes (see below). The remaining KDs are found at much higher values, usually above $3500 \mathrm{~cm}^{-1}$, and are probably not able to participate in the relaxation mechanism. A complete list of $g$ and $D$ values, their tensors, the excited states energies without $(\delta)$ and with $(\Delta)$ spin-orbit effects for complexes 1-4 can be found in Tables S11-S22. The orientation of the $g$ - and $D$-tensors, which is similar for each complex regardless the calculation method, is shown in Figure S14.

It has been stated that the sign and value of the $D$-parameter can be rationalized using the spin-orbit operator, which couples the ground and excited states. ${ }^{[2]}$ When the excited state results from the excitation between orbitals with the same $\left|m_{1}\right|$ values, the $\mathrm{MS}= \pm 3 / 2$ components are stabilized and a negative contribution to $D$ is obtained. On the other hand, an excitation

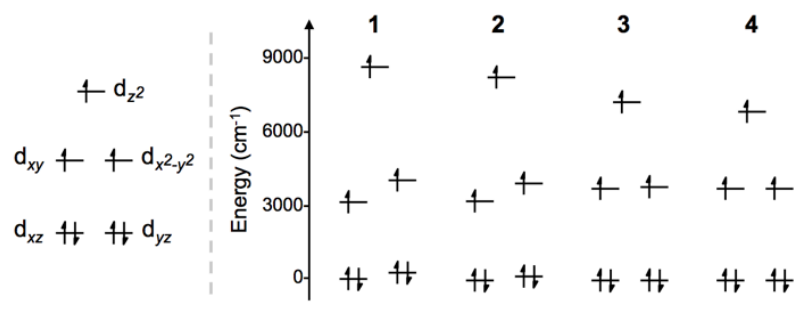

Figure 5. Schematic $d$ orbital splitting for a mononuclear $\mathrm{Co}^{\prime \prime}$ complex in a trigonal bipyramidal geometry (left) and AILF computed relative orbital energies and occupancies for complexes 1-4 (right).

between orbitals involving a $\left|\Delta m_{1}\right|=1$ change, i.e. stabilizing the MS $= \pm 1 / 2$ components, leads to a positive contribution to the $D$ value. $^{[5 b]}$ The expected $d$-orbital splitting for the trigonal bipyramidal Co" mononuclear complexes 1-4 is shown in Figure 5 ; the computed energies of the $d$ orbitals for each complex, which were obtained from the ab initio ligand field theory $(\text { AILFT })^{[25,26]}$ approach as implemented in ORCA, have also been included. As may be observed the electronic configuration of the quartets shown in Figure 5 indicate that the $D$ value should be positive because any excitation leading to an excited quartet state involves the transfer of an electron between

Table 2. ORCA/CASSCF calculated energies (in $\mathrm{cm}^{-1}$ ) and contributions to $D$ and $E$ for the first four excited states $\left({ }^{4} \Phi_{n}\right)$ of complexes 1-4.

\begin{tabular}{|c|c|c|c|c|}
\hline Complex & Excited state & Energy $\left(\mathrm{cm}^{-1}\right)$ & Cont. $D$ & Cont. $E$ \\
\hline \multirow{4}{*}{1} & ${ }^{4} \Phi_{1}$ & 3219.7 & -32.0 & 0.0 \\
\hline & ${ }^{4} \Phi_{2}$ & 4773.1 & 10.8 & -10.4 \\
\hline & ${ }^{4} \Phi_{3}$ & 5212.6 & 6.4 & 4.3 \\
\hline & ${ }^{4} \Phi_{4}$ & 5651.8 & 4.6 & 4.3 \\
\hline \multirow{5}{*}{2} & ${ }^{4} \Phi_{1}$ & 3215.2 & -30.9 & -0.2 \\
\hline & ${ }^{4} \Phi_{2}$ & 4620.0 & 10.5 & -10.6 \\
\hline & ${ }^{4} \Phi_{3}$ & 5047.0 & 7.3 & 5.2 \\
\hline & ${ }^{4} \Phi_{4}$ & 5360.1 & 4.7 & 4.0 \\
\hline & ${ }^{4} \Phi_{1}$ & 3692.7 & -30.9 & 0.0 \\
\hline \multirow{3}{*}{3} & ${ }^{4} \Phi_{2}$ & 4959.8 & 11.3 & -10.3 \\
\hline & ${ }^{4} \Phi_{3}$ & 5075.4 & 10.9 & 9.9 \\
\hline & ${ }^{4} \Phi_{4}$ & 6410.0 & 0.0 & 0.01 \\
\hline
\end{tabular}

\begin{tabular}{ccccc}
\multirow{4}{*}{4} & & 3619.6 & -30.5 & 0.0 \\
& ${ }^{4} \Phi_{1}$ & 4808.9 & 11.7 & -9.8 \\
& ${ }^{4} \Phi_{2}$ & 4808.9 & 11.7 & 9.8 \\
& ${ }^{4} \Phi_{3}$ & 5956.5 & 0.0 & 0.0 \\
\hline
\end{tabular}

orbitals possessing different $\left|m_{1}\right|$ values i.e. from $d_{x z}$ (or $d_{y z}$ ) to $d_{x y}$ (or $d_{x 2-y 2}$ ). This situation has already been reported for other penta-coordinated trigonal bipyramidal $\mathrm{Co}^{\prime \prime}$ complexes and the negative $D$ is usually attributed to the highly multiconfigurational character of the ground and low-lying excited states. ${ }^{[6,27]}$ Indeed, this can be confirmed by analyzing the $d$ orbital symmetry in the point group $C_{3 v}$; in this point group, two pairs of orbitals: $d_{x z} / d_{y z}$, and $d_{x y} / d_{x 2-y 2}$ belong to the same irreducible representation $E$, which enables their mixing and produces ground and excited states with significant contributions of several determinants (Table S23). It should be expected that the small negative $D$ values, found both by the experimental fit and the calculations, are obtained because the large negative contribution of the first excited state to the overall $D$ cannot be compensated by the smaller positive contributions of the higher energy excited states (Table 2 shows the energies and the contributions to $D$ and $E$ of the low-lying excited states found with ORCA/CASSCF methodology).

This fact can be explained by using complex 1 as an example. The first four excited states for this complex, denoted as ${ }^{4} \Phi_{\mathrm{n}}$, are close enough in energy to the ground state to have a significant impact on the magnitude of $D$ (Table 2). As stated above the ground state and the low-lying excited states are highly multideterminant (Table S23); Figure 6 shows the wave function compositions for the ground state $\left({ }^{4} \Phi_{0}\right)$ and the first excited state $\left({ }^{4} \Phi_{1}\right)$ of complex 1 . As may be observed the negative contribution to $D$ arises from the coupling between the $\mathrm{b}$ and $\mathrm{e}$ determinants of ${ }^{4} \Phi_{1}$ with the $c$ and $d$ determinants of ${ }^{4} \Phi_{0}$, because they involve excitations between orbitals having the same $m_{1}$ values. The interaction between the determinants $b$ $\left({ }^{4} \Phi_{1}\right)$ and e $\left({ }^{4} \Phi_{0}\right)$, which should imply an excitation with "double" negative contribution, may play a role as well.

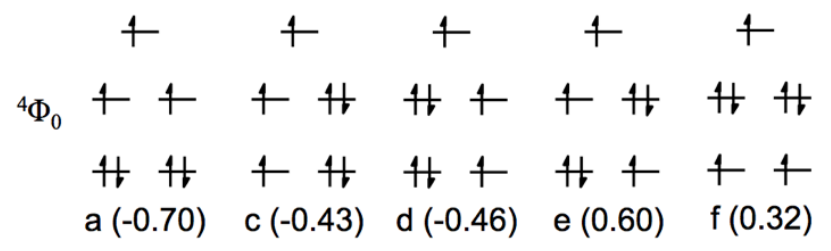

$$
\begin{aligned}
& +4 \\
& { }^{4} \Phi_{1}+4+4 \\
& \text { + H H } 4 \\
& \text { b (0.54) e (-0.83) }
\end{aligned}
$$

Figure 6. Composition of the wavefunctions corresponding to the ground state ${ }^{4} \Phi_{0}$ (top) and the first excited state ${ }^{4} \Phi_{1}$ (bottom) for complex 1 , including only the determinants that have the contributions larger than $5 \%$.

This approach can be also employed to confirm that the main interaction between the ground and the higher excited states leads to positive $D$ contributions. However, the large negative contribution of ${ }^{4} \Phi_{1}$ cannot be compensated by the smaller positive contributions of ${ }^{4} \Phi_{2},{ }^{4} \Phi_{3}$ and ${ }^{4} \Phi_{4}$ because those are located at higher energies. The same approach can be used to 
rationalize the negative sign of $D$ obtained for complexes $\mathbf{2 - 4}$, although in the case of the latter two complexes only the first three excited states show a significant contribution to the overall $D$ value.

The d orbital splitting obtained with the AILF method explains the differences in the magnitude of $D$ between both pairs of complexes with TPA (1-2) and tbta (3-4) ligands. As mentioned above, the presence of a chloride ligand should favor the stabilization of the equatorial $\left(\mathrm{d}_{\mathrm{xy}} / \mathrm{d}_{\mathrm{x} 2-\mathrm{y} 2}\right)$ orbital pair, thus producing a smaller energy gap between the occupied and the semioccupied d-orbitals and hence delivering a larger $D$ value for complexes 1 and 3. The computed energy gaps are 2796.3, $2929.5,3601.8$ and $3636.6 \mathrm{~cm}^{-1}$ for complexes 1-4, respectively, in a perfect agreement with what should be expected for both pairs of complexes (Table S24 contains a complete summary of the d-orbital splitting for each complex).

The computed relative energies of the lowest-lying KDs and the spin relaxation pathways of $\mathbf{1 - 4}$, computed with SIGLE_ANISO code implemented in MOLCAS, ${ }^{[28,29]}$ are shown in Figure 7 . In the case of complexes with TPA ligand (1-2) the spin relaxation mechanisms show a plausible pathway via a direct quantum tunneling (QTM) in the ground state. The matrix elements of the transition magnetic moments between states 1 - and 1+ are 0.45 and 0.49 for 1 and 2 , respectively, much higher than the 0.1 required value associated to an efficient relaxation mechanism. In addition, thermally assisted-QTM or Orbach processes seem very plausible for both compounds through their low-lying first KDs, which are found at 20.2 and $16.1 \mathrm{~cm}^{-1}$ for $\mathbf{1}$ and $\mathbf{2}$, respectively. The second excited KDs are much higher in energy (ca. $3200 \mathrm{~cm}^{-1}$ ) and therefore they are not expected to participate in the relaxation mechanism. The complexes containing the tbta ligand (3-4) show a slightly different behavior; in complex 3 the direct QTM and Orbach mechanisms are very
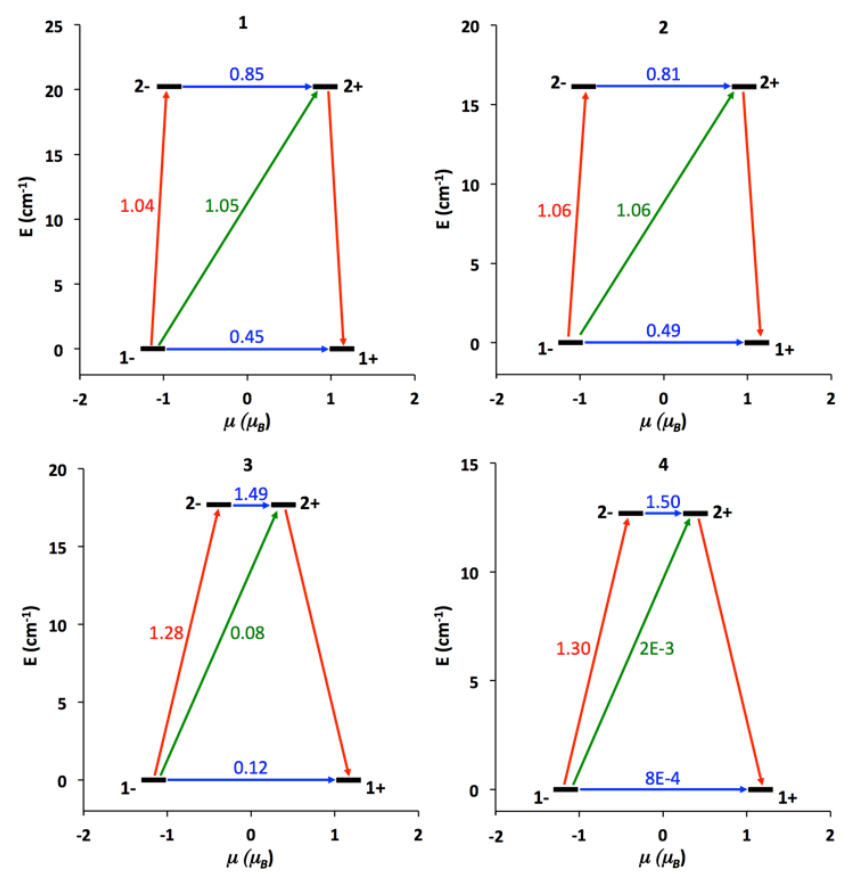

Figure 7. Lowest two Kramers' doublets and ab initio computed relaxation mechanism for complexes 1-4. The thick black lines imply KDs as a function of their magnetic moment along the main anisotropy axis. Red lines indicate the magnetization reversal mechanism. The blue lines correspond to ground state QTM and thermally assisted-QTM via the first excited KD, and green lines show possible Orbach relaxation processes. The values close to the arrows indicate the matrix elements of the transition magnetic moments (above 0.1 an efficient spin relaxation mechanism is expected). ${ }^{[4]}$

close to their operational limit although they may be still active. On the other hand the thermally assisted-QTM relaxation pathway seems very likely since the matrix elements for vertical excitation and tunneling at the first $\mathrm{KD}$, found $17.7 \mathrm{~cm}^{-1}$ above the ground state $K D$, are quite large. A similar situation is found in complex 4 although in this case the direct QTM and Orbach pathways seem to be completely shut down. The thermally assisted-QTM process is, however, plausible through the first excited KD, which is just $12.7 \mathrm{~cm}^{-1}$ higher in energy than the ground state. As above, the second excited KDs for $\mathbf{3}$ and $\mathbf{4}$ are much higher in energy (ca. $3600 \mathrm{~cm}^{-1}$ ) and are probably not involved in the spin relaxation process. As a whole, the computed barriers for the spin relaxation mechanism are very close to those obtained in the fitting of the magnetization data, with experimental and computed values of: 12 and $20.2 \mathrm{~cm}^{-1}$ for 1, 8.7 and $16.1 \mathrm{~cm}^{-1}$ for $2,8.1$ and $17.1 \mathrm{~cm}^{-1}$ for 3 , and 5 and $12.7 \mathrm{~cm}^{-1}$ for 4 .

\section{Conclusions}

In conclusion, the results show that slow magnetic relaxation can be observed under an applied dc field in the high spin penta-coordinate mononuclear Co" complexes with Ising-type magnetic anisotropy. This behaviour results from the stabilization of the largest $M_{s}= \pm 3 / 2$ components of the quartet state, which leads to an easy axis of magnetization in the presence of axial symmetry. The magnetic properties of these complexes were studied, and detailed ab initio calculations were performed to examine the anisotropy parameters and attain magneto-structural correlations. Besides reporting the SIMs with trigonal bipyramidal penta-coordinated Co" geometries, the present results also demonstrate the correlation between Isingtype magnetic anisotropy and structure in this kind of complexes.

\section{Experimental Section}

\section{Materials and Methods}

Magnetic measurements were performed using a Quantum Design SQUID-VSM magnetometer. The measured values were corrected for the experimentally measured contribution of the sample holder, while the derived susceptibilities were corrected for the diamagnetic contribution of the sample, estimated from Pascal's tables. ${ }^{[30]}$ Elemental analysis was performed on Elementar Microvario Cube Elemental Analyzer. IR spectrum was recorded on $\mathrm{KBr}$ pellets with a Perkin-Elmer spectrometer. Powder X-ray diffraction (PXRD) data was collected on a PANalytical EMPYREAN instrument using Cu-Ka radiation.

\section{X-ray Crystallography}

Intensity data were collected on a Brüker APEX-II CCD diffractometer using a graphite monochromated Mo-Ka radiation $(\alpha=0.71073 \AA)$. Data collection was performed using $\varphi$ and $\omega$ scan. The structure was solved using direct methods followed by full matrix least square refinements against $\mathrm{F}^{2}$ (all data HKLF 4 format) using SHELXTL. ${ }^{[31]}$ Subsequent 
difference Fourier synthesis and least-square refinement revealed the positions of the remaining non-hydrogen atoms. Determinations of the crystal system, orientation matrix, and cell dimensions were performed according to the established procedures. Lorentz polarization and multiscan absorption correction was applied. Non-hydrogen atoms were refined with independent anisotropic displacement parameters and hydrogen atoms were placed geometrically and refined using the riding model. All calculations were carried out using SHELXL 97, ${ }^{[32]}$ PLATON $99,{ }^{[33]}$ and WinGX systemVer-1.64. ${ }^{[34]}$ Crystallographic data for complexes 1-4 were summarized in Table S1.

\section{Synthesis of TPA (Tris(2-methylpyridyl)amine):}

A solution of $39.0 \mathrm{~g} \mathrm{(238} \mathrm{mmol)} \mathrm{of} \mathrm{2-picolyl} \mathrm{chloride} \mathrm{hydrochloride} \mathrm{in} 100$ $\mathrm{ml}$ of deionized water was cooled to $0^{\circ} \mathrm{C}$ in an ice bath. To this solution was added, with stirring, $45 \mathrm{ml}$ of a $5.3 \mathrm{~N}$ aqueous solution of $\mathrm{NaOH}$. The resulting free amine appeared as a bright red emulsion following the neutralization. To this mixture was then added a solution of $12.8 \mathrm{~g} \mathrm{(1} 19$ $\mathrm{mmol}$ ) of 2-(aminomethy1)pyridine in $150 \mathrm{ml}$ of dichloromethane. The mixture was then allowed to warm to room temperature and, for 2 days and an additional $45 \mathrm{ml}$ aliquot of $5.3 \mathrm{~N}$ aqueous $\mathrm{NaOH}$ solution was added. During addition of the $\mathrm{NaOH}$ solution, the $\mathrm{pH}$ of the aqueous portion of the reaction mixture was not allowed to exceed 9.5. The crude mixture was then washed with $100 \mathrm{ml}$ of $15 \% \mathrm{NaOH}$, and the organic phase was dried with $\mathrm{MgSO}_{4}$ and filtered. Removal of the dichloromethane yielded a brown solid mass which was extracted with boiling diethyl ether $(3 \times 50 \mathrm{ml})$. Evaporation of the ether extracts yielded yellow crystals of ligand. The ligand was purified by recrystallization from diethyl ether to give $27.2 \mathrm{~g}(80 \%)$ of white crystalline solid. ${ }^{1} \mathrm{H}$ NMR $\left(\mathrm{CDCl}_{3}\right): \delta 8.48$ (d), 7.69 (t), 7.60 (d), 7.17 (t), 3.81 (s). ${ }^{13} \mathrm{C} \mathrm{NMR}\left(\mathrm{CDCl}_{3}\right)$ : $\delta 160.51,149.85,137.25,123.74,122.94,60.83$.

\section{Synthesis of Tbta (Tris[(1-benzyl-1H-1,2,3-triazole-4} yl)methyl]amine):

Tripropargylamine $(13.2 \mathrm{~g}, 0.1 \mathrm{~mol})$ in acetonitrile $(100 \mathrm{ml})$ was treated sequentially with benzyl azide $(59.9 \mathrm{~g}, 0.45 \mathrm{~mol}), 2,6$-lutidine $(10.7 \mathrm{~g}, 0.1$ $\mathrm{mol})$, and $\mathrm{Cu}(\mathrm{MeCN})_{4} \mathrm{PF}_{6}(1.3 \mathrm{~mol} \%$ with respect to total alkyne). Upon addition of the copper salt, the reaction warmed and was cooled in an ice bath. After the mixture was stirred at room temperature for 4 days, a white crystalline solid precipitated from the reaction. Filtration and washing with cold acetonitrile gave white needle like crystals (43.8 g, $83 \%)$. The ligand was further purified by recrystallization from a hot $1: 1$ tert-butyl alcohol/water solution $(50 \mathrm{ml})$ followed by filtration and washing with water $(2 \times 30 \mathrm{ml})$. The white needle like crystals were dried under high vacuum overnight. Yield: $0.87 \mathrm{~g}, 87 \%$. Mp: $138-140{ }^{\circ} \mathrm{C} .{ }^{1} \mathrm{H}$ NMR $\left(\mathrm{CDCl}_{3}\right): \delta 3.70(\mathrm{~s}), 5.49(\mathrm{~s}), 7.26(\mathrm{~m}), 7.34(\mathrm{~m}), 7.67(\mathrm{~s}) .{ }^{13} \mathrm{C}$ NMR $\left(\mathrm{CDCl}_{3}\right): \delta 134.7,129.1,128.7,128.0,123.8,54.1,47.0$. Anal. Calcd for $\mathrm{C}_{30} \mathrm{H}_{30} \mathrm{~N}_{10}$ : C, 67.90; $\mathrm{H}, 5.70 ; \mathrm{N}, 26.40$. Found: $\mathrm{C}, 67.75 ; \mathrm{H}, 5.77 ; \mathrm{N}$, 26.33.

\section{Synthesis of $[\mathrm{Co}(\mathrm{TPA}) \mathrm{Cl}] \cdot \mathrm{ClO}_{4}(1)$ :}

TPA (29 mg, $0.1 \mathrm{mmol}$ ) was dissolved in $\mathrm{MeOH}(5 \mathrm{ml})$ and the solution was warmed to $40^{\circ} \mathrm{C}$. The mixture of $\mathrm{CoCl}_{2} \cdot 6 \mathrm{H}_{2} \mathrm{O}(12 \mathrm{mg}, 0.05 \mathrm{mmol})$ and $\mathrm{Co}\left(\mathrm{ClO}_{4}\right)_{2} \cdot 6 \mathrm{H}_{2} \mathrm{O}(18 \mathrm{mg}, 0.05 \mathrm{mmol})$ dissolved in $\mathrm{MeOH}(5 \mathrm{ml})$ was added dropwise to the above ligand solution while stirring. The resulting solution forms an intense red mixture that was stirred further for 30 minutes. The solution was then filtered off and the filtrate was left at open atmosphere for slow evaporation which yields large $\mathrm{X}$-ray quality red crystals of [Co(TPA)Cl] $\mathrm{ClO}_{4}$ (1) after 2 days. The crystals were separated, washed with cold water and $\mathrm{Et}_{2} \mathrm{O}$ and air-dried yield $(80 \%)$. Anal. Calcd for $\mathrm{C}_{25} \mathrm{H}_{27} \mathrm{CoN}_{5} \mathrm{O}_{6}$ : C, 54.36; $\mathrm{H}, 4.93 ; \mathrm{N}, 12.67 \%$. Found: $\mathrm{C}$, 54.45; H, 5.03; N, $12.73 \%$. IR (KBr pellet, $\left.4000-400 \mathrm{~cm}^{-1}\right) \mathrm{v} / \mathrm{cm}^{-1}$ : 3420, 3064, 2918, 2788, 1528, 1374, 1332, 1291, 1074, 1034.
TPA (29 mg, $0.1 \mathrm{mmol}$ ) was dissolved in $\mathrm{MeOH}(5 \mathrm{ml})$ and the solution was warmed to $40^{\circ} \mathrm{C}$. The mixture of $\mathrm{CoBr}_{2}(11 \mathrm{mg}, 0.05 \mathrm{mmol})$ and $\mathrm{Co}\left(\mathrm{ClO}_{4}\right)_{2} \cdot 6 \mathrm{H}_{2} \mathrm{O}(18 \mathrm{mg}, 0.05 \mathrm{mmol})$ dissolved in $\mathrm{MeOH}(5 \mathrm{ml})$ was added dropwise to the above ligand solution while stirring. The resulting solution forms an intense red mixture that was stirred further for 30 minutes. The solution was then filtered off and the filtrate was left at open atmosphere for slow evaporation which yields large $X$-ray quality red crystals of $[\mathrm{Co}(\mathrm{TPA}) \mathrm{Br}] \cdot \mathrm{ClO}_{4}$ (2) after 2 days. The crystals were separated, washed with cold water and $\mathrm{Et}_{2} \mathrm{O}$ and air-dried yield (55\%). Anal. Calcd for $\mathrm{C}_{27} \mathrm{H}_{29} \mathrm{CoN}_{7} \mathrm{O}_{6} \mathrm{~S}_{2}$ : C, 48.37; H, 4.36; N, $14.62 ; \mathrm{S}, 9.54 \%$. Found: C, 48.44; H, 4.44; N, 14.69; S, $9.61 \%$. IR (KBr pellet, $4000-400$ $\left.\mathrm{cm}^{-1}\right) \vee / \mathrm{cm}^{-1}: 3429,3052,2921,2838,2165,1525,1363,1327,1295$, 1070, 1052.

\section{Synthesis of $[\mathrm{Co}($ tbta $) \mathrm{Cl}] \cdot\left(\mathrm{ClO}_{4}\right) \cdot(\mathrm{MeCN})_{2} \cdot\left(\mathrm{H}_{2} \mathrm{O}\right)(3)$ :}

Tbta (53 mg, $0.1 \mathrm{mmol}$ ) was dissolved in MeCN (5 ml) and the solution was warmed to $40^{\circ} \mathrm{C}$. The mixture of $\mathrm{CoCl}_{2} \cdot 6 \mathrm{H}_{2} \mathrm{O}(12 \mathrm{mg}, 0.05 \mathrm{mmol})$ and $\mathrm{Co}\left(\mathrm{ClO}_{4}\right)_{2} \cdot 6 \mathrm{H}_{2} \mathrm{O}(18 \mathrm{mg}, 0.05 \mathrm{mmol})$ dissolved in $\mathrm{MeOH}(5 \mathrm{ml})$ was added dropwise to the above ligand solution while stiring. The resulting solution forms an intense red mixture that was stirred further for 30 minutes. The solution was then filtered off and the filtrate was left at open atmosphere for slow evaporation which yields large X-ray quality red crystals of $[\mathrm{Co}($ tbta $) \mathrm{Cl}] \cdot\left(\mathrm{ClO}_{4}\right) \cdot(\mathrm{MeCN})_{2} \cdot\left(\mathrm{H}_{2} \mathrm{O}\right)$ (3) after 3 days. The crystals were separated, washed with cold water and $\mathrm{Et}_{2} \mathrm{O}$ and air-dried yield (70 \%). Anal. Calcd for $\mathrm{C}_{26} \mathrm{H}_{29} \mathrm{ClCoN}_{6} \mathrm{O}_{10} \mathrm{~S}$ : C, 43.87; $\mathrm{H}, 4.11 ; \mathrm{N}$, 11.80; S, 4.49 \%. Found: C, 43.96; H, 4.21; N, 11.87; S, $4.55 \%$. IR ( $\mathrm{KBr}$ pellet, $\left.4000-400 \mathrm{~cm}^{-1}\right) \vee / \mathrm{cm}^{-1}: 3422,3073,2917,2818,2175,1529$, $1384,1318,1289,1076,1028,921$.

Synthesis of $[\mathrm{Co}(\mathrm{tbta}) \mathrm{Br}] \cdot \mathrm{ClO}_{4}(4)$ :

Tbta $(53 \mathrm{mg}, 0.1 \mathrm{mmol})$ was dissolved in $\operatorname{MeCN}(5 \mathrm{ml})$ and the solution was warmed to $40^{\circ} \mathrm{C}$. The mixture of $\mathrm{CoBr}_{2}(11 \mathrm{mg}, 0.05 \mathrm{mmol})$ and $\mathrm{Co}\left(\mathrm{ClO}_{4}\right)_{2} \cdot 6 \mathrm{H}_{2} \mathrm{O}(18 \mathrm{mg}, 0.05 \mathrm{mmol})$ dissolved in $\mathrm{MeOH}(5 \mathrm{ml})$ was added dropwise to the above ligand solution while stirring. The resulting solution forms an intense red mixture that was stirred further for 30 minutes. The solution was then filtered off and the filtrate was left at open atmosphere for slow evaporation which yields large $\mathrm{X}$-ray quality red crystals of $[\mathrm{Co}(\mathrm{tbta}) \mathrm{Br}] \cdot \mathrm{ClO}_{4}(4)$ after 3 days. The crystals were separated, washed with cold water and $\mathrm{Et}_{2} \mathrm{O}$ and air-dried yield (50\%). Anal. Calcd for $\mathrm{C}_{27} \mathrm{H}_{25} \mathrm{ClFeN}_{11} \mathrm{O}_{6}$ : C, 49.49; $\mathrm{H}, 3.84 ; \mathrm{N}, 23.50 \%$. Found: $\mathrm{C}, 49.58 ; \mathrm{H}$, 3.95; N, $23.56 \%$. IR ( $\mathrm{KBr}$ pellet, $\left.4000-400 \mathrm{~cm}^{-1}\right) \vee / \mathrm{cm}^{-1}: 3430,3064$, 2927, 2827, 2195, 1518, 1361, 1329, 1297, 1065, 1004, 875.

\section{Acknowledgements}

A.K.M. thanks UGC, India for the SRF fellowship. S.K. acknowledges DAE-BRNS (project no. No. 37(2)/14/09/2015BRNS), Government of India and IISER Bhopal for generous financial support. The research reported here was supported by the Spanish Ministerio de Economía y Competitividad (grant CTQ2015-64579-C3-1-P, MINECO/FEDER, UE). E.R. thanks Generalitat de Catalunya for an ICREA Academia award. J.J. and E.R. thankfully acknowledge the computer resources in the Consorci Serveis Universitaris de Catalunya (CSUC).

Keywords: Single Ion Magnets • trigonal bipyramidal geometry • - Ising-type anisotropy • ab initio calculations

\section{Reference}


[1] a) Caneschi, D. Gatteschi, R. Sessoli, A. L. Barra, L. C. Brunel, M Guillot, J. Am. Chem. Soc. 1991, 113, 5873; b) R. Sessoli, D. Gatteschi A. Caneschi, M. A. Novak, Nature 1993, 365, 141; c) C. Delfs, D. Gatteschi, L. Pardi, R. Sessoli, K. Wieghardt, D. Hanke, Inorg. Chem 1993, 32, 3099; d) R. Sessoli, H. L. Tsai, A. R. Schake, S. Y. Wang, J. B. Vincent, K. Folting, D. Gatteschi, G. Christou, D. N. Hendrickson, J. Am. Chem. Soc. 1993, 115, 1804; e) D. Gatteschi, A. Caneschi, L. Pardi, R. Sessoli, Science 1994, 265, 1054; f) A. L. Barra, A. Caneschi, A. Cornia, F. F. de Biani, D. Gatteschi, C. Sangregorio, R. Sessoli, L. Sorace, J. Am. Chem. Soc. 1999, 121, 5302; g) E. K. Brechin, J. Yoo, M. Nakano, J. C. Huffman, D. N. Hendrickson, G. Christou, Chem Commun. 1999, 783; h) S. Maheswaran, G. Chastanet, S. J. Teat, T. Mallah, R. Sessoli, W. Wernsdorfer, R. E. P. Winpenny, Angew. Chem., Int. Ed. 2005, 44, 5044

[2] a) M. N. Leuenberger, D. Loss, Nature 2001, 410, 789; b) L. Bogani, W. Wernsdorfer, Nat. Mater. 2008, 7, 179.

[3] a) R. J. Blagg, C. A. Muryn, E. J. L. Mclnnes, F. Tuna, R. E. P. Winpenny, Angew. Chem., Int. Ed. 2011, 50,6530; b) J. D. Rinehart, M. Fang, W. J. Evans, J. R. Long, Nat. Chem. 2011, 3, 538; c) J. D. Rinehart, M. Fang, W. J. Evans, J. R. Long, J. Am. Chem. Soc. 2011, 133, 14236; d) J. J. Le Roy, L. Ungur, I. Korobkov, L. F. Chibotaru, M. Murugesu, J. Am. Chem. Soc. 2014, 136, 8003; e) L. Ungur, J. J. Le Roy, I. Korobkov, M. Murugesu, L. F. Chibotaru, Angew. Chem., Int. Ed. 2014, 53, 4413; f) L. J. Batchelor, I. Cimatti, R. Guillot, F. Tuna, W. Wernsdorfer, L. Ungur, L. F. Chibotaru, V. E. Campbell, T. Mallah Dalton Trans. 2014, 43, 12146; g) V. E. Campbell, H. Bolvin, E. Rivière, R. Guillot, W. Wernsdorfer, T. Mallah, Inorg. Chem. 2014, 53, 2598; h) S.-D. Jiang, B.-W. Wang, H.-L. Sun, Z.-M. Wang, S. Gao, J. Am. Chem Soc. 2011, 133, 4730; i) A. K. Mondal, S. Goswami, S. Konar, Dalton Trans. 2015, 44, 5086; j) D. N. Woodruff, R. E. P. Winpenny, R. A Layfield, Chem. Rev. 2013, 113, 5110; k) J. D. Rinehart, J. R. Long Chem. Sci. 2011, 2, 2078; I) F. Habib, M. Murugesu, Chem. Soc. Rev. 2013, 42, 3278; m) P. Zhang, Y. N. Guo, J. Tang, Coord. Chem. Rev. 2013, 257, 1728; n) S. Goswami, A. K. Mondal, S. Konar, Inorg. Chem. Front. 2015, 2, 687; o) S. K. Gupta, T. Rajeshkumar, G. Rajaraman, R. Murugavel, Chem. Sci. 2016, 7, 5181; p) A. J. Brown, D. Pinkowicz, M. R. Saber, K. R. Dunbar, Angew. Chem. Int. Ed. 2015, 54, 5864; q) J. Liu, Y.-C. Chen, J.-L. Liu, V. Vieru, L. Ungur, J.-H. Jia, L. F. Chibotaru, Y. Lan, W. Wernsdorfer, S. Gao, X.-M. Chen, M.-L. Tong, J. Am. Chem. Soc. 2016, 138, 5441; r) Y.-C. Chen, J.-L. Liu, L. Ungur, J. Liu, Q.-W. Li L.-F. Wang, Z.-P. Ni, L. F. Chibotaru, X.-M. Chen, M.-L. Tong, J. Am. Chem. Soc. 2016, 138, 2829; s) Y.-S. Meng, S.-D. Jiang, B.-W. Wang, S. Gao, Acc. Chem. Res. 2016, 49, 2381.

[4] a) W. H. Harman, T. D. Harris, D. E. Freedman, H. Fong, A. Chang, J. D. Rinehart, A. Ozarowski, M. T. Sougrati, F. Grandjean, G. J. Long, J. R. Long, C. J. Chang, J. Am. Chem. Soc. 2010, 132, 18115; b) D. Weismann, Y. Sun, Y. Lan, G. Wolmershäuser, A. K. Powell, H. Sitzmann, Chem. - Eur. J. 2011, 17, 4700; c) P. H. Lin, N. C. Smythe, S I. Gorelsky, S. Maguire, N. J. Henson, I. Korobkov, B. L. Scott, J. C. Gordon, R. T. Baker, M. Murugesu, J. Am. Chem. Soc. 2011, 133, 15806; d) S. Mossin, B. L. Tran, D. Adhikari, M. Pink, F. M. Heinemann, J. Sutter, R. K. Szilagyi, K. Meyer, D. J. Mindiola, J. Am Chem. Soc 2012, 134, 13651; e) J. M. Zadrozny, M. Atanasov, A. M. Bryan, C. Y. Lin, B. D. Rekken, P. P. Power, F. Neese, J. R. Long, Chem. Sci. 2013, 4, 125; f) T. Jurca, A. Farghal, P. H. Lin, I. Korobkov, M. Murugesu, D. S. Richardson, J. Am. Chem. Soc. 2011, 133, 15814; g) J. M. Zadrozny, J. Liu, N. A. Piro, C. J. Chang, S. Hill, J. R. Long, Chem. Commun. 2012, 48, 3927; h) J. Vallejo, I. Castro, R. R. García, J. Cano, M. Julve, F. Lloret, G. D. Munno, W. Wernsdorfer, E. Pardo, J. Am. Chem. Soc. 2012, 134, 15704; i) R. Ruamps, R. Maurice, L. Batchelor, M. BoggioPasqua, R. Guillot, A. L. Barra, J. J. Liu, E. Bendeif, S. Pillet, S. Hill, T. Mallah, N. Guihery, J. Am. Chem. Soc. 2013, 135, 3017; j) S. GómezCoca, E. Cremades, N. Aliaga-Alcalde, E. Ruiz, Inorg. Chem. 2014, 53, 676; k) G. A. Craig, M. Murrie, Chem. Soc. Rev. 2015, 44, 2135; I) S Gómez-Coca, D. Aravena, R. Morales, E. Ruiz, Coord. Chem. Rev. 2015, 289, 379; m) A. K. Mondal, J. Jover, E. Ruiz, S. Konar, Chem. Commun. 2017, 53, 5338.
[5] a) Y. Y. Zhu, C. Cui, Y. Q. Zhang, J. H. Jia, X. Guo, C. Gao, K. Qian, S D. Jiang, B. W. Wang, Z. M. Wang, S. Gao, Chem. Sci. 2013, 4, 1802; b) S. Gómez-Coca, E. Cremades, N. Aliaga- Alcalde, E. Ruiz, J. Am. Chem. Soc. 2013, 135, 7010; c) F. Yang, Q. Zhou, Y. Zhang, G. Zeng, G. Li, Z. Shi, B. Wang, S. Feng, Chem. Commun. 2013, 49, 5289; d) D. Wu, X. Zhang, P. Huang, W. Huang, M. Ruan, Z. W. Ouyang, Inorg Chem. 2013, 52, 10976; e) W. Huang, T. Liu, D. Wu, J. Cheng, Z. W. Ouyang, C. Duan, Dalton Trans. 2013, 42, 15326; f) R. Herchel, L. Váhovská, I. Potočňák, Z. Trávníček, Inorg. Chem. 2014, 53, 5896; g) C. Mathonière, H.-J. Lin, D. Siretanu, R. Clérac, J. M. Smith, J. Am. Chem. Soc. 2013, 135, 19083; h) X. Feng, C. Mathonière, I.-R. Jeon, M. Rouzières, A. Ozarowski, M. L. Aubrey, M. I. Gonzalez, R. Clérac, J. R. Long, J. Am. Chem. Soc. 2013, 135, 15880; i) R. Ruamps, L. J. Batchelor, R. Guillot, G. Zakhia, A.-L. Barra, W. Wernsdorfer, N. Guihéry, T. Mallah, Chem. Sci. 2014, 5, 3418; j) F. Shao, B. Cahier, N. Guihéry, E. Rivière, R. Guillot, A.-L. Barra, Y. Lan, W. Wernsdorfer, V. E. Campbell, T. Mallah, Chem. Commun. 2015, 51, 16475.

[6] a) J. M. Zadrozny, D. J. Xiao, M. Atanasov, G. J. Long, F. Grandjean, F. Neese, J. R. Long, Nat. Chem. 2013, 5, 577; b) R. C. Poulten, M. J. Page, A. G. Algarra, J. J. Le Roy, I. Lopez, E. Carter, A. Llobet, S. A. Macgregor, M. F. Mahon, D. M. Murphy, M. Murugesu, M. K. Whittlesey, J. Am. Chem. Soc. 2013, 135, 13640; c) Y.-Z. Zhang, S. Gómez-Coca, A. J. Brown, M. R. Saber, X. Zhang, K. R. Dunbar, Chem. Sci. 2016, 7, 6519; d) R. Boča, J. Miklovič, J. Titiš, Inorg. Chem. 2014, 53, 2367; e) D. Brazzolotto, M. Gennari, S. Yu, J. Pécaut, M. Rouzières, R. Clérac, M. Orio, C. Duboc, Chem. Eur. J. 2016, 22, 925; f) X.-N. Yao, J.-Z. Du, Y.-Q. Zhang, X.-B. Leng, M.-W. Yang, S.-D. Jiang, Z.-X. Wang, Z.-W. Ouyang, L. Deng, B.-W. Wang, S. Gao, J. Am. Chem. Soc. 2017, 139, 373; g) T. J. Woods, M. F. Ballesteros-Rivas, S. Gómez-Coca, E. Ruiz, K. R. Dunbar, J. Am. Chem. Soc. 2016, 138, 16407; h) D. Schweinfurth, M. G. Sommer, M. Atanasov, S. Demeshko, S. Hohloch, F. Meyer, F. Neese, B. Sarkar, J. Am. Chem. Soc. 2015, 137, 1993; i) F. Shao, B. Cahier, E. Rivière, R. Guillot, N. Guihéry, V. E. Campbell, T. Mallah, Inorg. Chem. 2017, 56, 1104; j) C. Rajnák, J. Titiš, O. Fuhr, M. Ruben, R. Boča, Inorg. Chem. 2014, 53, 8200; k) M. R. Saber, K. R. Dunbar, Chem. Commun. 2014, 50, 12266; I) S. Vaidya, A. Upadhyay, S. K. Singh, T. Gupta, S. Tewary, S. K. Langley, J. P. S. Walsh, K. S. Murray, G. Rajaraman, M. Shanmugam, Chem. Commun. 2015, 51, 3739; m) L. Smolko, J. Černák, M. Dušek, J. Miklovič, J. Titiš, R. Boča, Dalton Trans. 2015, 44, 17565.

[7] a) A. Caneschi, D. Gatteschi, N. Lalioti, C. Sangregorio, R. Sessoli, G. Venturi, A. Vindigni, A. Rettori, M. G. Pini, M. A. Novak, Angew. Chem. Int. Ed. 2001, 40, 1760; b) M. Murrie, Chem. Soc. Rev. 2010, 39, 1986.

[8] H. A. Kramers, Proc. R. Acad. Sci. Amsterdam 1930, 33, 959.

[9] A. W. Addison, T. N. Rao, J. Reedijk, J. van Rijn, G. C. Verschoor, J. Chem. Soc., Dalton Trans. 1984, 1349.

[10] M. Jevric, T. Zheng, N. K. Meher, J. C. Fettinger, M. Mascal, Angew. Chem., Int. Ed. 2011, 50, 717.

[11] F. E. Mabbs, D. J. Machin, Magnetism and Transition Metal Complexes; Dover Publications: Mineola, NY, 2008.

[12] N. F. Chilton, R. P. Anderson, L. D. Turner, A. Soncini, K. S. Murray, J. Comput. Chem. 2013, 34, 1164.

[13] a) C. Benelli, D. Gatteschi, Inorg. Chem. 1982, 21, 1788; b) A. Bencini, C. Benelli, D. Gatteschi, C. Zanchini, Inorg. Chem. 1980, 19, 3839; c) I. Bertini, D. Gatteschi, A. Scozzafava, Inorg. Chem. 1975, 14, 812; d) C. Benelli, D. Gatteschi, G. Speroni, Inorg. Chim. Acta. 1984, 90, 179.

[14] D. Gatteschi, R. Sessoli, J. Villain, Molecular Nanomagnets; Oxford University Press: Oxford, U.K. 2006.

[15] a) K. S. Cole, R. H. Cole, J. Chem. Phys. 1941, 9, 341; b) Y.-N. Guo, G.-F. Xu, Y. Guo, J. Tang, Dalton Trans. 2011, 40, 9953

[16] a) S. Xue, Y. N. Guo, L. Zhao, P. Zhang, J. Tang, Dalton Trans. 2014, 43, 1564; b) P. H. Lin, T. J. Burchell, R. Clérac, M. Murugesu, Angew. Chem., Int. Ed. 2008, 47, 8848; c) G. F. Xu, Q. L. Wang, P. Gamez, Y. Ma, R. Clérac, J. Tang, S. P. Yan, P. Cheng, D. Z. Liao, Chem. Commun. 2010, 46, 1506; d) M. T. Gamer, Y. Lan, P. W. Roesky, A. K. Powell, R. Clérac, Inorg. Chem. 2008, 47, 6581; e) S.-Y. Lin, C. Wang, L. Zhao, J. Tang, Chem. Asian J. 2014, 9, 3558; f) S.-Y. Lin, L. Zhao, Y.-N. Guo, P. Zhang, Y. Guo, J. Tang, Inorg. Chem. 2012, 51, 10522; 
g) R. J. Blagg, L. Ungur, F. Tuna, J. Speak, P. Comar, D. Collison, W. Wernsdorfer, E. J. L. Mclnnes, L. F. Chibotaru, R. E. P. Winpenny, Nat. Chem. 2013, 5, 673; h) R. J. Blagg, F. Tuna, E. J. L. Mclnnes, R. E. P. Winpenny, Chem. Commun. 2011, 47, 10587; i) K. Qian, X.-C. Huang, C. Zhou, X.-Z. You, X.-Y. Wang, K. R. Dunbar, J. Am. Chem. Soc. 2013 135, 13302.

[17] a) C.-S. Liu, M. Du, E. Carolina Sañudo, J. Echevarría, M. Hu, Q. Zhang, L.-M. Zhou, S.-M. Fang, Dalton Trans. 2011, 40, 9366; b) E. Colacio, J. Ruiz-Sánchez, F. J. White, E. K. Brechin, Inorg. Chem. 2011, 50, 7268; c) J. M. Zadrozny, J. R. Long, J. Am. Chem. Soc. 2011, 133, 20732.

[18] R. Orbach, Proc. R. Soc. London, Ser. A 1961, 264, 458

[19] K. R. Meihaus, J. R. Rinehart, J. R. Long, Inorg. Chem. 2011, 50, 8484

[20] F. Neese, WIREs Comput. Mol. Sci. 2012, 2, 73 .

[21] G. Karlström, R. Lindh, P.-Å. Malmqvist, B. O. Roos, U. Ryde, V. Veryazov, P.-O. Widmark, M. Cossi, B. Schimmelpfennig, P. Neogrady, L. Seijo, Comput. Matter Sci. 2003, 28, 222.

[22] V. Veryazov, P.-O. Widmark, L. Serrano-Andrés, R. Lindh, B. O. Roos, Int. J. Quantum Chem. 2004, 100, 626.

[23] F. Aquilante, L. De Vico, N. Ferré, G. Ghigo, P.-A. Malmqvist, P. Neogrády, T. B. Pedersen, M. Pitoňák, M. Reiher, B. O. Roos, L. Serrano-Andrés, M. Urban, V. Veryazov, R. Lindh, J. Comp. Chem. 2010, 31, 224

[24] B. A. Heß, C. M. Marian, U. Wahlgren, O. Gropen, Chem. Phys. Lett., 1996, 251, 365.

[25] M. Atanasov, D. Ganyushin, K. Sivalingam, F. Neese, In Structure and Bonding, D. M. P. Mingos, P. D. a. J. P. D., Ed. Springer: Berlin, 2012 149.

[26] M. Atanasov, D. Aravena, E. Suturina, E. Bill, D. Maganas, F. Neese, Coord. Chem. Rev. 2015, 289, 177.

[27] R. Ruamps, L. J. Batchelor, R. Maurice, N. Gogoi, P. Jiménez-Lozano, N. Guihéry, C. de Graaf, A.-L. Barra, J.-P. Sutter, T. Mallah, Chem. Eur. J. 2013, 19, 950.

[28] L. F. Chibotaru, L. Ungur, A. Soncini, Angew. Chem. Int. Ed. 2008, 47, 4126.

[29] L. F. Chibotaru, L. Ungur, C. Aronica, H. Elmoll, G. Pillet, D. Luneau, J. Am. Chem. Soc. 2008, 130, 12445.

[30] O. Kahn, Molecular Magnetism, VCH Publishers Inc. 1991

[31] G. M. Sheldrick, SHELXTL Program for the Solution of Crystal of Structures, University of Göttingen, Göttingen, Germany, 1993.

[32] G. M .Sheldrick, SHELXL 97, Program for Crystal Structure Refinement, University of Göttingen, Göttingen, Germany, 1997.

[33] A. L. Spek, J Appl. Crystallogr. 2003, 36, 7.

[34] L. J. Farrugia, J. Appl. Crystallogr. 1999, 32, 837. 


\section{Entry for the Table of Contents}

\section{FULL PAPER}

A quantitative estimation of magnetic anisotropy has been done for $\mathrm{C}_{3}$ symmetric $\mathrm{Co}^{\prime \prime}$ complexes with Isingtype magnetic anisotropy by using a combined experimental and theoretical approach.

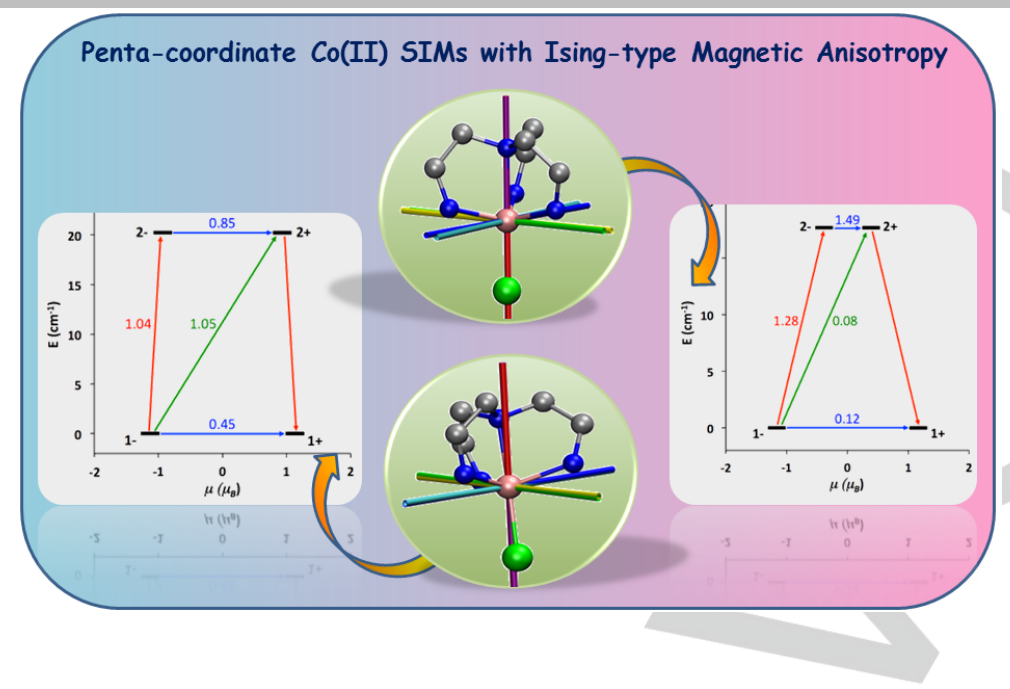

Single Ion Magnets

Amit Kumar Mondal, Jesús Jover, Eliseo Ruiz* and Sanjit Konar*

m - $\mathbf{\square}$

Quantitative

Estimation of Isingtype Magnetic

Anisotropy in a Family of $\mathrm{C}_{3}$

Symmetric Co" Complexes 